\title{
Sublattice effect on topological surface states in complex $(\mathrm{SnTe})_{n>1}\left(\mathrm{Bi}_{2} \mathrm{Te}_{3}\right)_{m=1}$ compounds
}

\author{
S. V. Eremeev, ${ }^{1,2,3}$ T. V. Menshchikova, ${ }^{2}$ I. V. Silkin, ${ }^{2}$ M. G. Vergniory, ${ }^{4}$ P. M. Echenique, ${ }^{4,5}$ and E. V. Chulkov ${ }^{2,3,4,5}$ \\ ${ }^{1}$ Institute of Strength Physics and Materials Science, 634021, Tomsk, Russia \\ ${ }^{2}$ Tomsk State University, 634050 Tomsk, Russia \\ ${ }^{3}$ Saint Petersburg State University, 198504 Saint Petersburg, Russia \\ ${ }^{4}$ Donostia International Physics Center (DIPC), 20018 San Sebastián/Donostia, Basque Country, Spain \\ ${ }^{5}$ Departamento de Física de Materiales UPV/EHU, Centro de Física de Materiales CFM - MPC and Centro Mixto CSIC-UPV/EHU, \\ 20080 San Sebastián/Donostia, Basque Country, Spain
}

(Received 31 March 2015; revised manuscript received 1 June 2015; published 19 June 2015)

\begin{abstract}
An exotic type of topological spin-helical surface state was found in layered van der Waals bonded $(\mathrm{SnTe})_{n=2,3}\left(\mathrm{Bi}_{2} \mathrm{Te}_{3}\right)_{m=1}$ compounds which comprise two covalently bonded band inverted sublattices, SnTe and $\mathrm{Bi}_{2} \mathrm{Te}_{3}$, within a building block. This topological state demonstrates unusual dispersion within the band gap. The dispersion of the surface state has two linear sections of different slope with a shoulder feature between them. Such a dispersion of the topological surface state enables an effective switch of the velocity of topological carriers by means of applying an external electric field.
\end{abstract}

DOI: 10.1103/PhysRevB.91.245145

PACS number(s): 85.75.-d, 71.20.Nr, 73.20.At

\section{INTRODUCTION}

Topological insulators (TIs) are materials with a gap in the bulk electronic spectrum driven by the spin-orbit interaction (SOI), which is topologically distinct from ordinary insulators. This distinction, characterized by a $\mathbb{Z}_{2}$ topological invariant, is manifested in the existence of the time-reversal-symmetry protected spin-helical gapless surface state having linear (or almost linear) Dirac dispersion.

Starting from the discovery of the TI phase in the quintuple layer (QL) structured binary compounds $\mathrm{Bi}_{2} \mathrm{Te}_{3}, \mathrm{Bi}_{2} \mathrm{Se}_{3}$, and $\mathrm{Sb}_{2} \mathrm{Te}_{3}$ and its thorough experimental and theoretical investigation [1-9], a huge number of $\mathbb{Z}_{2}$ TIs were found. Most of the known TIs are $\mathrm{Bi}_{2} \mathrm{Te}_{3}$-type ternary and quaternary layered materials. Among them QL and septuple layer (SL) structured compounds as well as materials with alternating QL and SL units were theoretically predicted and experimentally confirmed [10-13].

Another class of topological materials consists of materials with the band gap inverted by strong spin-orbit interaction in which the topological surface states are protected by crystal mirror symmetry [14] --so-called topological crystalline insulators (TCIs). One example of a TCI is SnTe $[15,16]$, showing an even number of the Dirac cones in the surface spectrum.

Recently it was demonstrated that in the $\mathrm{Bi}_{2} \mathrm{Te}_{3} / \mathrm{SnTe}$ heterostructure, the system containing $\mathbb{Z}_{2}$ and crystal-mirrorsymmetry TIs, the $\bar{\Gamma}$ Dirac surface states of $\mathrm{Bi}_{2} \mathrm{Te}_{3}$ and $\mathrm{SnTe}$ annihilate at the interface while the Dirac state of SnTe at the $\bar{M}$ "survives" [17]. This points out that the interaction of $\mathbb{Z}_{2}$ TI and TCI systems can results in unusual changes in the topological surface state picture.

Different systems containing layers of band-inverted $\mathrm{Bi}_{2} \mathrm{Te}_{3}$ and SnTe materials are $(\mathrm{SnTe})_{n}\left(\mathrm{Bi}_{2} \mathrm{Te}_{3}\right)_{m}$ compounds [18]. In this series the $(n=1, m>1)$ compounds- $\mathrm{SnBi}_{4} \mathrm{Te}_{7}$, $\mathrm{SnBi}_{6} \mathrm{Te}_{10}$, and $\mathrm{SnBi}_{8} \mathrm{Te}_{13}$-are structurally identical to earlier studied lead-based TIs [10], containing alternating QL and SL structural units, while the $(n \geqslant 1, m=1)$ compounds are formed by unique, nonalternating building blocks. The simplest system $(n=1, m=1)$, i.e., $\mathrm{SnBi}_{2} \mathrm{Te}_{4}$ is composed of SL hexagonally ordered blocks stacked along the $c$ axis and separated by van der Waals spacings. The SL building block [Fig. 1(b)] can be obtained from the original QL block of $\mathrm{Bi}_{2} \mathrm{Te}_{3}$ [Fig. 1(a)] by introducing the SnTe bilayer between the $\mathrm{Bi}$ atomic layer and the central $\mathrm{Te}$ one. This compound was predicted to be three-dimensional (3D) $\mathbb{Z}_{2}$ TI with single Dirac cone in the bulk energy gap [10]. The $\mathrm{Sn}_{2} \mathrm{Bi}_{2} \mathrm{Te}_{5}$ compound $(n=2, m=1)$ is formed by nonuple layer (NL) building blocks [18]. The NL block [Fig. 1(c)] is constructed from a SL block by introduction of an additional SnTe hexagonal bilayer in the middle of the SL or two bilayers into the original QL. Thus this compound differs from the $\mathrm{Bi}_{2} \mathrm{Te}_{3} / \mathrm{SnTe}$ heterostructure having ionic-covalent bonded $\mathrm{Bi}_{2} \mathrm{Te}_{3}$ and $\mathrm{SnTe}$ atomic layers. Another experimentally confirmed structure $(\mathrm{SnTe})_{n}\left(\mathrm{Bi}_{2} \mathrm{Te}_{3}\right)_{m}$, which is also built from ionic-covalent bonded $\mathrm{Bi}_{2} \mathrm{Te}_{3}$ and $\mathrm{SnTe}$ atomic layers, is $\mathrm{Sn}_{3} \mathrm{Bi}_{2} \mathrm{Te}_{6}(n=3, m=1)$ [18]. In this case additional $\mathrm{SnTe}$ layers are incorporated into the building block [Fig. 1(d)]. We are not aware of the possibility of growth of stable $(\mathrm{SnTe})_{n}\left(\mathrm{Bi}_{2} \mathrm{Te}_{3}\right)_{m=1}$ compounds with larger $n$ which have building blocks of the same type.

In the present paper we demonstrate by means of relativistic density functional theory (DFT) calculations that $(\mathrm{SnTe})_{n=2,3}\left(\mathrm{Bi}_{2} \mathrm{Te}_{3}\right)_{m=1}$ compounds are $\mathbb{Z}_{2}$ TIs in which, owing to complex band inversion of bulk states belonging to $\mathrm{Bi}_{2} \mathrm{Te}_{3}$ and $\mathrm{SnTe}$ sublattices at the surface, a topological surface state (TSS) arises demonstrating dog-leg dispersion within the bulk energy gap. We discuss the localization and spin texture of the TSS in the context of gate control of the topological transport properties. We also demonstrate the existence of such a TSS in $\mathrm{Sn}_{2} \mathrm{Sb}_{2} \mathrm{Te}_{5}$ compound.

\section{METHODS}

For calculations we use the Vienna Ab Initio Simulation Package (VASP) $[19,20]$ with the generalized gradient approximation (GGA) [21] for the exchange correlation potential. The interaction between the ion cores and valence electrons was described by the projector augmented-wave method $[22,23]$. Relativistic effects, including spin-orbit interaction (SOI), were taken into account. The atomic positions of 
(a)

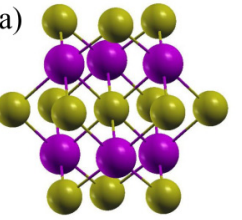

(b)

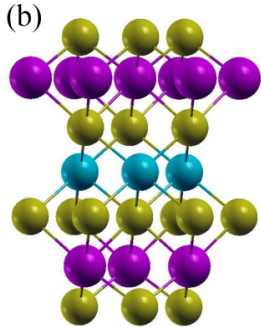

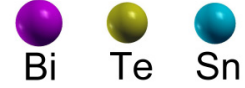

(c)

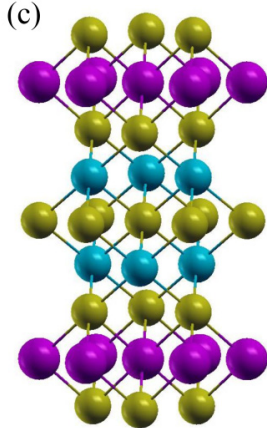

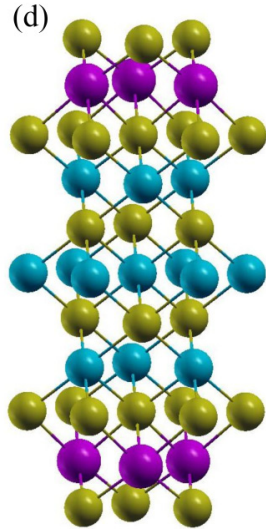

FIG. 1. (Color online) Building blocks of $\mathrm{Bi}_{2} \mathrm{Te}_{3}$ (a), $\mathrm{SnBi}_{2} \mathrm{Te}_{4}$ (b), $\mathrm{Sn}_{2} \mathrm{Bi}_{2} \mathrm{Te}_{5}$ (c), and $\mathrm{Sn}_{3} \mathrm{Bi}_{2} \mathrm{Te}_{6}(\mathrm{~d})$.

bulk ( $\mathrm{SnTe})_{n=2,3}\left(\mathrm{Bi}_{2} \mathrm{Te}_{3}\right)$ compounds were optimized. In slab calculations a vacuum space of $\sim 20 \AA$ was included to ensure negligible interaction between opposite surfaces. $k$-point meshes of $7 \times 7 \times 7$ and $9 \times 9 \times 1$ were used for the bulk and slab calculations, respectively. The total-energy convergence was better than $1.0 \times 10^{-6} \mathrm{eV}$. To additionally examine the validity of our results, complementary calculations for bulk and surface band structures of $\mathrm{Sn}_{2} \mathrm{Bi}_{2} \mathrm{Te}_{5}$ were performed using the full-potential linearized augmented plane-wave method as implemented in the FLEUR code [24]. We compared the results with those obtained from the VASP calculations and found very good agreement between the two sets of data.

\section{RESULTS AND DISCUSSION}

The bulk band structures of $\mathrm{Sn}_{2} \mathrm{Bi}_{2} \mathrm{Te}_{5}$ shown in Fig. 2 have been calculated with and without SOI included. As can be seen in Fig. 2(a), a direct energy gap of $0.32 \mathrm{eV}$ exists at the $\Gamma$ point in the calculation without SOI, which transforms into an indirect gap of $0.11 \mathrm{eV}$ after switching on the SOI [Fig. 2(b)], which is in a good agreement with the experimental value of $0.09 \mathrm{eV}$ [18]. We also examined the effect of nonlocal van der Waals ( $\mathrm{vdW}$ ) interaction on structure optimization and bulk electronic structure of $\mathrm{Sn}_{2} \mathrm{Bi}_{2} \mathrm{Te}_{5}$, taking it into account by the Grimme method as implemented in the VASP code. We found that vdW corrections do not change the indirect bulk band gap, and just slightly modify the gap in the Brillouin zone center, increasing it by $35 \mathrm{meV}$.

The time-reversal-invariant momenta (TRIMs) in this Brillouin zone are the $\Gamma, \mathrm{A}$, and triply degenerate $\mathrm{M}$ and $\mathrm{L}$ points. We checked the parity of the wave functions at all TRIMs to determine the topological character of this material and found that the parity inversion of bulk bands occurs at the $\Gamma$ point which leads to $\mathbb{Z}_{2}$ invariant $1 ;(000)$ as in $\mathrm{Bi}_{2} \mathrm{Te}_{3}$.

In spite of this similarity in SOI-induced wave function parity change, the total band inversion at $\Gamma$ is an entanglement of two different inversions corresponding to each sublattice due to the complex crystal structure of the compound. For instance, in $\mathrm{Bi}_{2} \mathrm{Te}_{3}$ the SOI modifies the top valence band state (predominantly of $p_{z}$ character) of Te atoms lying in
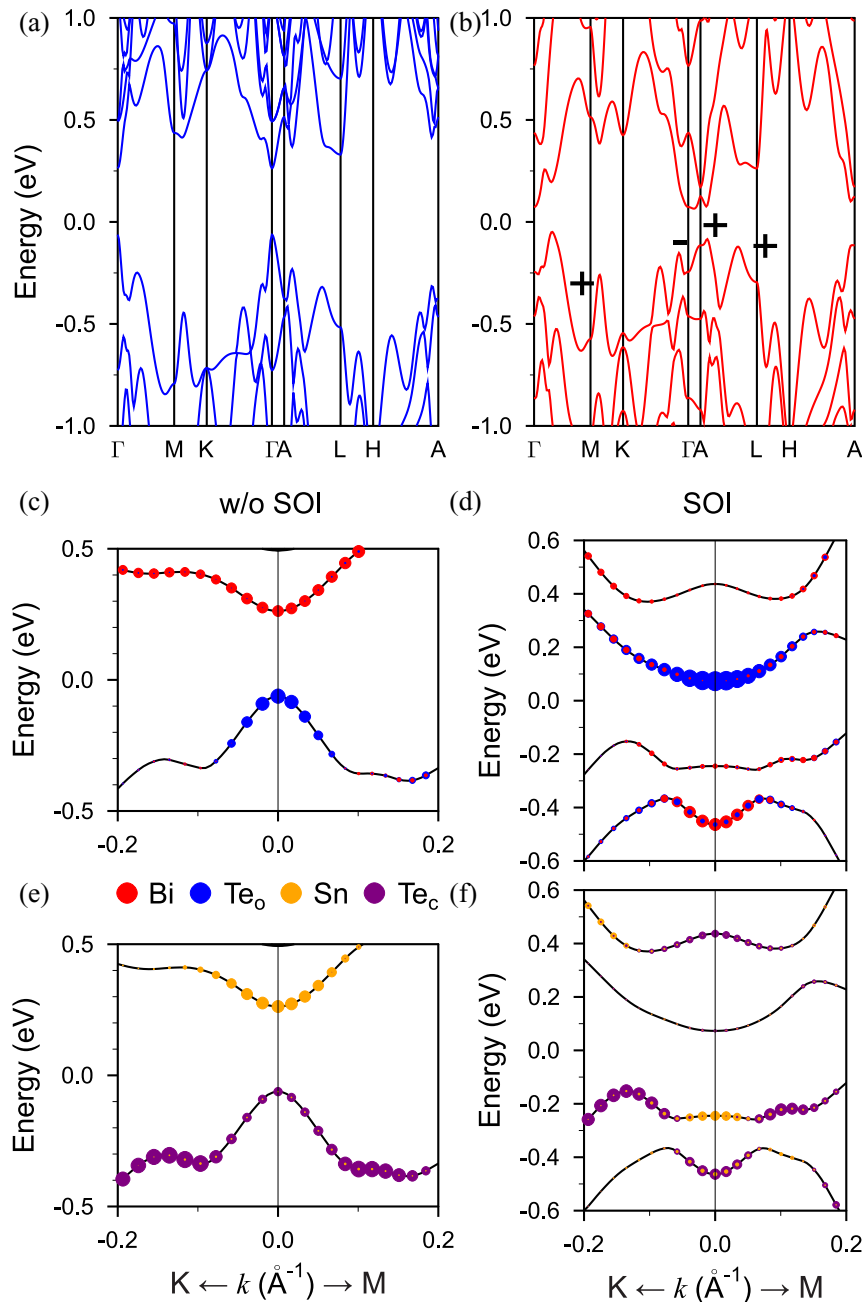

(d)
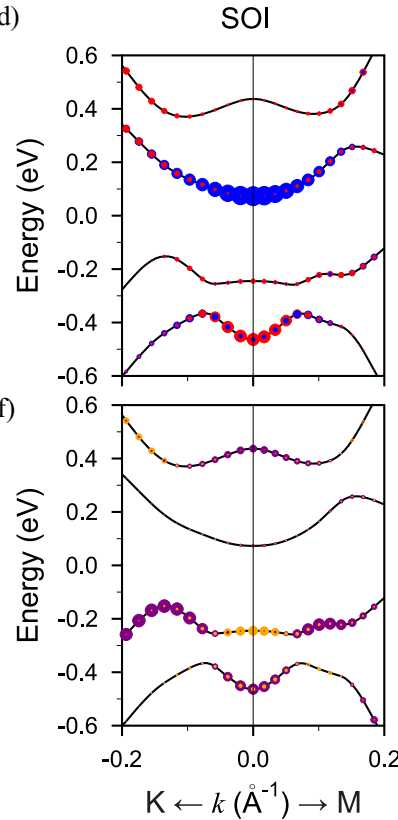

FIG. 2. (Color online) Bulk electronic structure of $\mathrm{Sn}_{2} \mathrm{Bi}_{2} \mathrm{Te}_{5}$ calculated without (a) and with (b) SOI included (Signs of band parities at the TRIM are also shown). Atomic composition of the near-gap states in bulk $\mathrm{Sn}_{2} \mathrm{Bi}_{2} \mathrm{Te}_{5}$ calculated without (c,d) and with $(\mathrm{e}, \mathrm{f}) \mathrm{SOI}$ included.

the outer layers of the $\mathrm{QL}\left(\mathrm{Te}_{\mathrm{o}}\right)$ and the $\mathrm{Bi} p_{z}$ state of the bottom conduction band so that they are inverted in the close vicinity of the $\Gamma$ point. A similar simple band inversion occurs in bulk SnTe where $\mathrm{Sn}$ and Te states are inverted in the $\mathrm{L}$ point of the cubic Brillouin zone. Instead, in $\mathrm{Sn}_{2} \mathrm{Bi}_{2} \mathrm{Te}_{5}$ the spectra calculated without $\mathrm{SOI}$ show that both $\mathrm{Bi}$ and $\mathrm{Sn}$ states form the bottom of the conduction band, while the states of $\mathrm{Te}_{\mathrm{o}}$ atoms and tellurium atoms of the central layer of the $\mathrm{NL}\left(\mathrm{Te}_{\mathrm{c}}\right)$ lie in the top valence band [Figs. 2(c) and 2(d)]. Switching on the SOI results in an inversion of $\mathrm{Bi}$ and $\mathrm{Te}_{\mathrm{o}}$ states and $\mathrm{Sn}$ and $\mathrm{Te}_{\mathrm{c}}$ states. However, in contrast to the binary systems this inversion has a more complex character. As can be seen in Fig. 2(e), after switching on the $\mathrm{SOI}$ the $\mathrm{Te}_{\mathrm{o}}$ states become dominant in the conduction band while the $\mathrm{Bi}$ states (in contrast to the $\mathrm{Bi}_{2} \mathrm{Te}_{3}$ case) occupy the second valence band in the vicinity of $\Gamma$. The SOI-inverted Sn states lie in the highest valence band and $\mathrm{Te}_{\mathrm{c}}$ states appear in the second conduction band Fig. 2(f). Additionally, the SOI-inverted Sn states (with smaller weight) appear in the second valence band. Thus, the $\mathrm{Sn}_{2} \mathrm{Bi}_{2} \mathrm{Te}_{5} \mathrm{TI}$ has a competitive bulk $\Gamma$-gap band inversion which comprises 


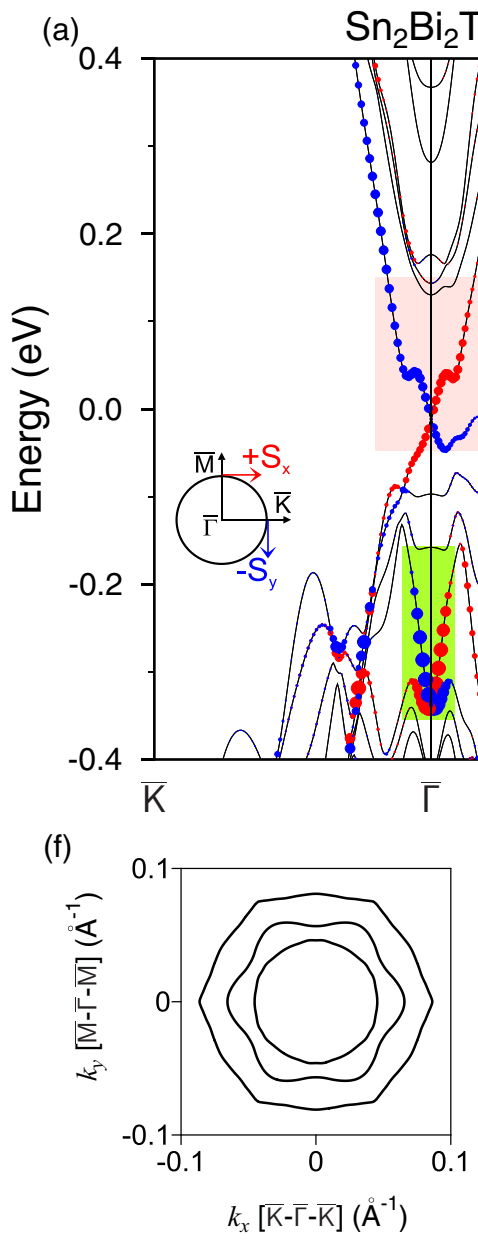

(b)

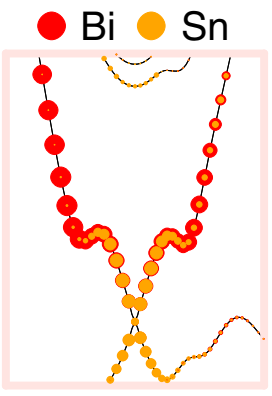

(c)

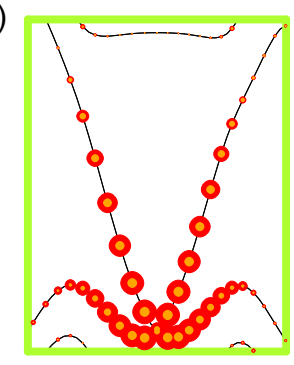

(d)

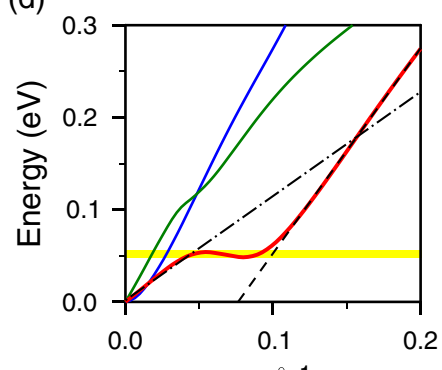

$k_{\|}\left(\AA^{-1}\right)$

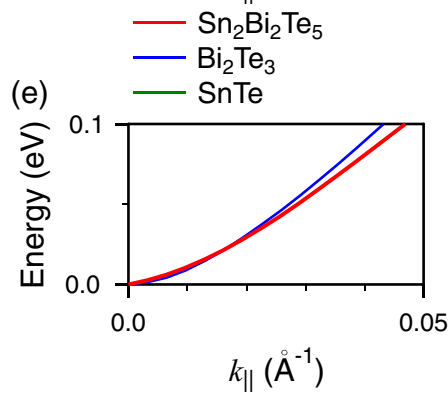

(h)

(g)

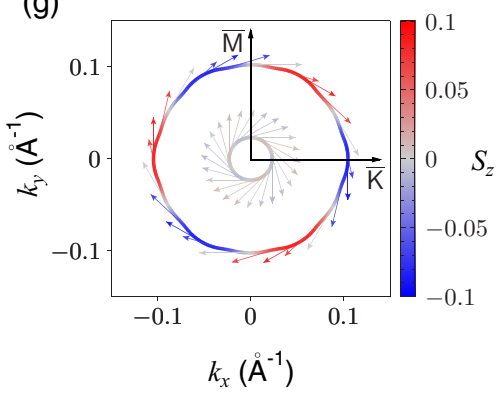

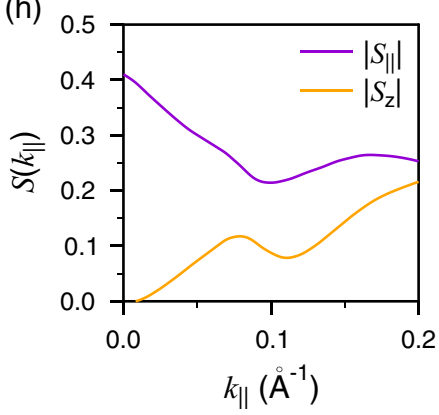

FIG. 3. (Color online) (a) Surface electronic structure of $\mathrm{Sn}_{2} \mathrm{Bi}_{2} \mathrm{Te}_{5}$. The sizes of the colored circles represent the weight of the states in the outermost building block (NL) of the slab; red and blue colors show the sign of the in-plane $\left(S_{x}\right.$ and $\left.S_{y}\right)$ spin components; light pink and light green rectangles mark the areas of the band-gap and valence-band topological surface states, respectively. Atomic weights of Bi and $\mathrm{Sn}$ in the band-gap (b) and valence-band (c) TSSs. (d) Energy dispersion of the band-gap TSS compared to $\mathrm{Bi}_{2} \mathrm{Te}_{3}$ and $\mathrm{SnTe}_{\bar{\Gamma}} \mathrm{Dirac}$ cones. Dashed and dot-dashed lines are linear fits for the TSS above and below the shoulder, respectively. (e) Dispersion of the valence-band TSS compared to $\mathrm{Bi}_{2} \mathrm{Te}_{3}$. (f) Constant energy contours in the shoulder region [yellow stripe in panel (d)]. (g) Spin-resolved constant energy contours for band-gap TSS at energies of $25 \mathrm{meV}$ below and above the shoulders. Arrows adjacent to the contours denote the in-plane spin component $S_{\|}$. The out-of-plane spin component $S_{z}$ is indicated by the color, with red and blue corresponding to positive and negative values, respectively. (h) The spin components $S_{\|}$and $S_{z}$ for band-gap TSS as functions of $k_{\|}$along the $\bar{\Gamma}-\overline{\mathrm{K}}$ direction.

the inversion of the states belonging to different sublattices, SnTe and $\mathrm{Bi}_{2} \mathrm{Te}_{3}$, so that the valence band edge is mainly formed by $\mathrm{Sn}$ states and the conduction band edge occupied by $\mathrm{Te}_{\mathrm{o}}$ states, while the $\mathrm{Bi}$ and $\mathrm{Te}_{\mathrm{c}}$ states lie next to the edges bands.

Such a superposition of the SOI-induced bulk band inversions in the the $\mathrm{SnTe}$ and $\mathrm{Bi}_{2} \mathrm{Te}_{3}$ sublattices of the $(\mathrm{SnTe})_{2}\left(\mathrm{Bi}_{2} \mathrm{Te}_{3}\right)_{1}$ TI results in formation of unusual topological states on the surface. In the surface spectrum two topological surface states arise at the $\bar{\Gamma}$ point. The upper TSS lies in the band gap [see the light pink rectangle in Fig. 3(a)] while the lower TSS (light green rectangle in Fig. 3(a)] resides in the valence band (band-gap and valence-band TSS's, respectively, from now on). The lower, valence-band TSS, propagating within the local valence band gap, has typical Dirac dispersion for TIs. In contrast, the band-gap TSS displays nonlinear dog-leg behavior. It has two linear sections in the spectrum-near the band crossing (Dirac point) and below the conduction band-which connect to each other in the middle part of the gap with formation of shoulders in the spectrum. As in other TIs, the TSSs in $\mathrm{Sn}_{2} \mathrm{Bi}_{2} \mathrm{Te}_{5}$ are localized within the outermost building block of the slab. However, in this case, where the building block has two band-inverted SnTe and $\mathrm{Bi}_{2} \mathrm{Te}_{3}$ sublattices, the localization of the TSS is affected by the peculiarities in the bulk band inversion. Figures 3(b) and 3(c) show the Bi and Sn atomic weights in the TSSs. As one can see in Fig. 3(c), the valence-band TSS has dominant $\mathrm{Bi}$ weights that reflect its dominant localization in the outer $\mathrm{Bi}$-Te layers of the NL. Comparing the dispersion of this state with that of the Dirac cone in $\mathrm{Bi}_{2} \mathrm{Te}_{3}$ [8] [Fig. 3(e)] one can conclude that at small $k_{\|}$both bands are almost identical. In the band-gap TSS [Fig. 3(b)], the lower linear section near the band crossing, the Sn contribution dominates and thus the state is mainly localized in the inner Sn-Te layers 
of the NL. Approaching the shoulders the Sn contribution disappears rather quickly, and above the band shoulders the TSS becomes mostly localized in the outer Bi-Te layers. Thus the band shoulders in the TSS spectrum are associated with the change of the TSS localization from the SnTe to $\mathrm{Bi}_{2} \mathrm{Te}_{3}$ sublattice. It should be noted that the slope of the upper linear section of the band-gap TSS (at $k_{\|}>0.1 \AA^{-1}$ ) localized in the outer $\mathrm{Bi}$-Te layers is comparable with the slope of the $\mathrm{Bi}_{2} \mathrm{Te}_{3}$ Dirac cone [Fig. 3(d)], while the dispersion of the lower linear section $\left(k_{\|}<0.05 \AA^{-1}\right)$ differs considerably from both $\mathrm{Bi}_{2} \mathrm{Te}_{3}$ and rocksalt-SnTe [25] $\bar{\Gamma}$ Dirac states. As a consequence, the linear fitting for lower and upper sections of the band-gap TSS [dot-dashed and dashed lines in Fig. 3(d)] gives $E / k_{\|}$ slopes of 1.13 and 2.22, respectively. Between these linear sections, in the shoulder region at $0.05<k_{\|}<0.1 \AA^{-1}$, two inflection points exist which limit the almost flat section in the TSS spectrum. Such a very low velocity regime in the band-gap TSS resembles the heavy-fermion topological state that has been proposed theoretically [26] and obtained from magnetothermoelectric transport measurements [27] in the topological Kondo insulator (TKI) $\mathrm{SmB}_{6}$. Owing to slightly negative dispersion of the TSS in the shoulder region [see the narrow yellow stripe in Fig. 3(d)], by varying the chemical potential the system will pass from one velocity mode in the lower linear section to the other velocity mode in the upper linear section through the three surface Fermi pockets regime at energy $\approx 50 \mathrm{meV}$ [Fig. 3(f)].

The detailed spin texture of the band-gap TSS is illustrated in Figs. 3(g) and 3(h). Figure 3(g) shows spin-resolved constant energy contours (CECs) for the lower (inner contour) and upper (outer contour) linear sections of the TSS. Both contours, apart from the in-plane clockwise spin polarization, also demonstrate the presence of the out-of-plane spin component, which is an intrinsic feature of spin-polarized states at hexagonal surfaces, while $S_{z}$ for the inner contour is extremely small. As clearly seen for the outer contour, $S_{z}$ is zero along $\bar{\Gamma}-\overline{\mathrm{M}}$ and reaches a minimum (maximum) value in $\bar{\Gamma}-\overline{\mathrm{K}}$ directions. The dependencies of absolute values for $S_{\|}$and $S_{z}$ on $k_{\|}$along $\bar{\Gamma}-\overline{\mathrm{K}}$ are shown in Fig. 3(h). The in-plane spin polarization having a maximum near the band crossing point rapidly decreases approaching the shoulder. At $k_{\|}>0.1 \AA^{-1}$, i.e., in the upper linear section, it increases with $k_{\|}$until it reaches the region of the bulk states. In contrast, the out-of-plane spin polarization increases with $k_{\|}$in both linear sections of the TSS, having a kink at the band shoulder.

The increase of the SnTe layers in the building block of $(\mathrm{SnTe})_{n}\left(\mathrm{Bi}_{2} \mathrm{Te}_{3}\right)_{m=1}$ compounds up to $n=3$ maintains the two TSSs in the surface spectrum as well as the dog-leg behavior in the dispersion of the band-gap surface state [Fig. 4(a)]. The difference from the $\mathrm{Sn}_{2} \mathrm{Bi}_{2} \mathrm{Te}_{5}$ case is that the band shoulders lie closer to the bulk valence band, making the lower section of the TSS very short and two times less steep.

Another way to modify the parameters of the band-gap TSS is substitution of Bi by Sb. In this case SnTe layers are introduced into the $\mathrm{Sb}_{2} \mathrm{Te}_{3}$ TI forming a $(\mathrm{SnTe})_{n}\left(\mathrm{Sb}_{2} \mathrm{Te}_{3}\right)_{m=1}$ compound. As can be seen in Fig. 4(b) the surface spectrum of $\mathrm{Sn}_{2} \mathrm{Sb}_{2} \mathrm{Te}_{5}$ also holds two TSSs, with dog-leg dispersion in the band-gap TSS. However, in contrast to the previous case the band shoulders arise near the conduction band.
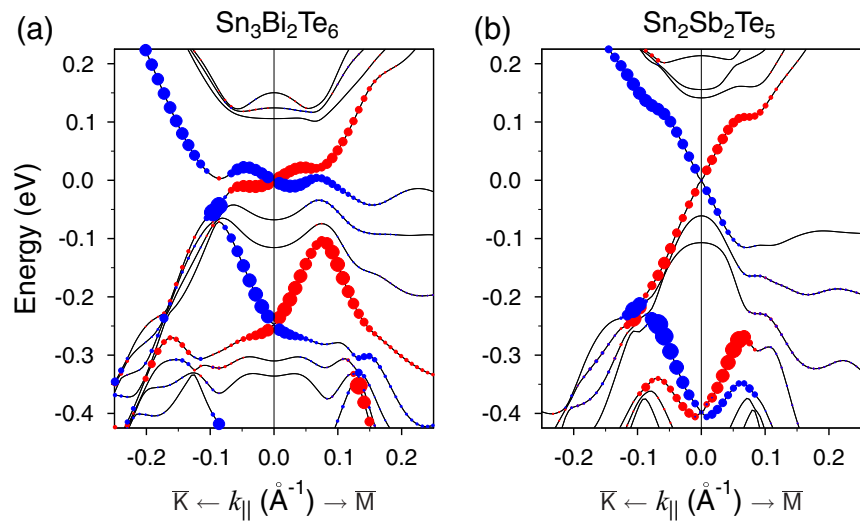

FIG. 4. (Color online) Spin-resolved surface electronic structure of $\mathrm{Sn}_{3} \mathrm{Bi}_{2} \mathrm{Te}_{6}$ (a) and $\mathrm{Sn}_{2} \mathrm{Sb}_{2} \mathrm{Te}_{5}$ (b).

\section{SUMMARY}

In summary, on the basis of ab initio DFT calculations we have demonstrated that $(\mathrm{SnTe})_{n=2,3}\left(\mathrm{Bi}_{2} \mathrm{Te}_{3}\right)_{m=1}$ compounds are $\mathbb{Z}_{2}$ topological insulators in which, owing to superposition of two pairs of SOI-induced band inversions realized in $\mathrm{SnTe}$ and $\mathrm{Bi}_{2} \mathrm{Te}_{3}$ sublattices, two spin-helical topological surface states arise at the $\bar{\Gamma}$ point. These states reside in the band gap and in the local gap of the valence band. The latter TSS, lying deep below the Fermi level, has a dispersion similar to that in the $\mathrm{Bi}_{2} \mathrm{Te}_{3} \mathrm{TI}$, while the band-gap TSS demonstrates exotic dog-leg dispersion with two linear sections of different slope and a very low velocity shoulder section between them. In $\mathrm{Sn}_{2} \mathrm{Bi}_{2} \mathrm{Te}_{5}$ the shoulders are located in the middle of the gap, and velocities of carriers in lower and upper linear sections differ by a factor of 2 . Thus, in contrast to all hitherto known TIs, where the slope of the spin-helical TSS is linear or varies smoothly with energy, the band-gap TSS in $(\mathrm{SnTe})_{n=2,3}\left(\mathrm{Bi}_{2} \mathrm{Te}_{3}\right)_{m=1}$ compounds shows an abrupt switch of the velocity of topological carriers with change in the Fermi surface topology in the narrow energy range (shoulder section) where spin and charge current should be blocked. We also demonstrated that similar TSSs exist in $(\mathrm{SnTe})_{n=2}\left(\mathrm{Sb}_{2} \mathrm{Te}_{3}\right)_{m=1}$ compound. This finding enhance the understanding of the diversity of topological surface states and paves a way for the efficient control of the group velocity with sufficiently large spin current density by tuning the chemical potential.

Further tuning of the band-gap TSS parameters can be realized by using $\left(\mathrm{Bi}_{1-x} \mathrm{Sb}_{x}\right)_{2} \mathrm{Te}_{3}[28,29]$ or $\mathrm{Bi}_{2}\left(\mathrm{Te}_{1-x} \mathrm{Se}_{x}\right)_{3}$ $[29,30]$ compounds as basic TIs for construction of $(\mathrm{SnTe})_{n}\left((\mathrm{Bi}-\mathrm{Sb})_{2}(\mathrm{Te}-\mathrm{Se})_{3}\right)_{m=1}$ systems.

\section{ACKNOWLEDGMENTS}

We acknowledge partial support from the Basque Country Government, Departamento de Educación, Universidades e Investigación (Grant No. IT-756-13), the Spanish Ministerio de Ciencia e Innovación (Grant No. FIS2010-19609-C02-01), the Tomsk State University Academic D.I. Mendeleev Fund Program (Grant No. 8.1.05.2015), the Russian Foundation for Basic Research (Grant No. 13-02-12110-ofi-m) and Saint Petersburg State University (project 11.50.202.2015). Numerical calculations were performed on the SKIF-Cyberia supercomputer at the National Research Tomsk State University. 
[1] Y. Xia, D. Qian, D. Hsieh, L. Wray, A. Pal, H. Lin, A. Bansil, D. Grauer, Y. S. Hor, R. J. Cava, and M. Z. Hasan, Nat. Phys. 5, 398 (2009).

[2] Y. L. Chen, J. G. Analytis, J.-H. Chu, Z. K. Liu, S.-K. Mo, X. L. Qi, H. J. Zhang, D. H. Lu, X. Dai, Z. Fang, S. C. Zhang, I. R. Fisher, Z. Hussain, and Z.-X. Shen, Science 325, 178 (2009).

[3] Tong Zhang, Peng Cheng, Xi Chen, Jin-Feng Jia, Xucun Ma, Ke He, Lili Wang, Haijun Zhang, Xi Dai, Zhong Fang, Xincheng Xie, and Qi-Kun Xue, Phys. Rev. Lett. 103, 266803 (2009).

[4] K. Kuroda, M. Arita, K. Miyamoto, M. Ye, J. Jiang, A. Kimura, E. E. Krasovskii, E. V. Chulkov, H. Iwasawa, T. Okuda, K. Shimada, Y. Ueda, H. Namatame, and M. Taniguchi, Phys. Rev. Lett. 105, 076802 (2010).

[5] H. Zhang, C.-X. Liu, X.-L. Qi, X. Dai, Z. Fang, and S.-C. Zhang, Nat. Phys. 5, 438 (2009).

[6] W. Zhang, W. Yu, H. J. Zhang, X. Dai, and Z. Fang, New J. Phys. 12, 065013 (2010).

[7] Y. Zhang, K. He, C.-Z. Chang, C.-L. Song, L.-L. Wang, X. Chen, J.-F. Jia, Z. Fang, X. Dai, W.-Y. Shan, S.-Q. Shen, Q. Niu, X.-L. Qi, S.-C. Zhang, X.-C. Ma, and Q.-K. Xue, Nat. Phys. 6, 584 (2010).

[8] S. V. Eremeev, Yu. M. Koroteev, E. V. Chulkov, JETP Lett. 91, 387 (2010).

[9] K. Eto, Z. Ren, A. A. Taskin, K. Segawa, and Y. Ando, Phys. Rev. B 81, 195309 (2010).

[10] S. V. Eremeev, G. Landolt, T. V. Menshchikova, B. Slomski, Y. M. Koroteev, Z. S. Aliev, M. B. Babanly, J. Henk, A. Ernst, L. Patthey, A. Eich, A. A. Khajetoorians, J. Hagemeister, O. Pietzsch, J. Wiebe, R. Wiesendanger, P. M. Echenique, S. S. Tsirkin, I. R. Amiraslanov, J. H. Dil, and E. V. Chulkov, Nat. Commun. 3, 635 (2012).

[11] K. Kuroda, H. Miyahara, M. Ye, S. V. Eremeev, Yu. M. Koroteev, E. E. Krasovskii, E. V. Chulkov, S. Hiramoto, C. Moriyoshi, Y. Kuroiwa, K. Miyamoto, T. Okuda, M. Arita, K. Shimada, H. Namatame, M. Taniguchi, Y. Ueda, and A. Kimura, Phys. Rev. Lett. 108, 206803 (2012).

[12] M. Neupane, S.-Y. Xu, L. A. Wray, A. Petersen, R. Shankar, N. Alidoust, Chang Liu, A. Fedorov, H. Ji, J. M. Allred, Y. S. Hor,
T.-R. Chang, H.-T. Jeng, H. Lin, A. Bansil, R. J. Cava, and M. Z. Hasan, Phys. Rev. B 85, 235406 (2012).

[13] T. Okuda, T. Maegawa, M. Ye, K. Shirai, T. Warashina, K. Miyamoto, K. Kuroda, M. Arita, Z. S. Aliev, I. R. Amiraslanov, M. B. Babanly, E. V. Chulkov, S. V. Eremeev, A. Kimura, H. Namatame, and M. Taniguchi, Phys. Rev. Lett. 111, 206803 (2013).

[14] L. Fu, Phys. Rev. Lett. 106, 106802 (2011).

[15] T. H. Hsieh, H. Lin, J. Liu, W. Duan, A. Bansil, and L. Fu, Nat. Commun. 3, 982 (2012).

[16] Y. Tanaka, Z. Ren, K. Nakayama, S. Souma, T. Takahashi, K. Segawa, and Y. Ando, Nat. Phys. 8, 800 (2012).

[17] T. Rauch, M. Flieger, J. Henk, and I. Mertig, Phys. Rev. B 88, 245120 (2013).

[18] B. A. Kuropatwa and H. Kleinke, Z. Anorg. Allg. Chem. 638, 2640 (2012).

[19] G. Kresse and J. Hafner, Phys. Rev. B 48, 13115 (1993).

[20] G. Kresse and J. Furthmüller, Comput. Mater. Sci. 6, 15 (1996).

[21] J. P. Perdew, K. Burke, and M. Ernzerhof, Phys. Rev. Lett. 77, 3865 (1996).

[22] P. E. Blöchl, Phys. Rev. B 50, 17953 (1994).

[23] G. Kresse and D. Joubert, Phys. Rev. B 59, 1758 (1999).

[24] http://www.flapw.de

[25] S. V. Eremeev, Yu. M. Koroteev, I. A. Nechaev, and E. V. Chulkov, Phys. Rev. B 89, 165424 (2014).

[26] V. Alexandrov, M. Dzero, and P. Coleman, Phys. Rev. Lett. 111, 226403 (2013).

[27] Yongkang Luo, Hua Chen, Jianhui Dai, Zhu-an Xu, and J. D. Thompson, Phys. Rev. B 91, 075130 (2015).

[28] J. Zhang, C.-Z. Chang, Z. Zhang, J. Wen, X. Feng, K. Li, M. Liu, K. He, L. Wang, X. Chen, Q.-K. Xue, X. Ma, and Y. Wang, Nat. Commun. 2, 574 (2011).

[29] Z. Ren, A. A. Taskin, S. Sasaki, K. Segawa, and Y. Ando, Phys. Rev. B 84, 165311 (2011).

[30] A. M. Shikin, I. I. Klimovskikh, S. V. Eremeev, A. A. Rybkina, M. V. Rusinova, A. G. Rybkin, E. V.Zhizhin, J. Sanchez-Barriga, A. Varykhalov, I. P. Rusinov, E. V. Chulkov, K. A. Kokh, V. A. Golyashov, V. Kamyshlov, and O. E. Tereshchenko, Phys. Rev. B 89, 125416 (2014). 